Grand Valley State University

ScholarWorks@GVSU

$5-2000$

\title{
Cultural and Developmental Comparisons of Landscape Perceptions and Preferences
}

Thomas R. Herzog

Grand Valley State University, herzogt@gvsu.edu

Eugene J. Herbert

Rachel Kaplan

University of Michigan - Ann Arbor

C. L. Crooks

Grand Valley State University

Follow this and additional works at: https://scholarworks.gvsu.edu/psy_articles

Part of the Psychology Commons

\section{ScholarWorks Citation}

Herzog, Thomas R.; Herbert, Eugene J.; Kaplan, Rachel; and Crooks, C. L., "Cultural and Developmental Comparisons of Landscape Perceptions and Preferences" (2000). Peer Reviewed Articles. 44.

https://scholarworks.gvsu.edu/psy_articles/44

This Article is brought to you for free and open access by the Psychology Department at ScholarWorks@GVSU. It has been accepted for inclusion in Peer Reviewed Articles by an authorized administrator of ScholarWorks@GVSU. For more information, please contact scholarworks@gvsu.edu. 


\section{CULTURAL AND DEVELOPMENTAL COMPARISONS OF LANDSCAPE PERCEPTIONS AND PREFERENCES}

THOMAS R. HERZOG is a professor of psychology at Grand Valley State University in Allendale, Michigan. His current research focuses on environmental preferences, restorative environments, and the psychology of humor.

EUGENE J. HERBERT is a landscape architect and team leader of the Conservation and Land Management section of Warringah Council's Environmental Management unit in Sydney, Australia. His interest lies in the practical design and land management applications of landscape preference and perception theory.

RACHEL KAPLAN is a professor of environmental psychology at the School of Natural Resources and Environment and professor in the Department of Psychology at the University of Michigan. Her various areas of research in environmental psychology are reflected in her recent book (with S. Kaplan \& R. L. Ryan), With People in Mind: Design and Management of Everyday Nature.

C. L. CROOKS received a bachelor's degree in psychology from Grand Valley State University and is currently a graduate student in psychology at the University of Georgia.

The authors compared several Australian subgroups and American college students on their preferences for Australian natural landscapes. Preference correlations across groups were generally high, with the correlations for Australian adults somewhat lower. Factor analysis yielded six perceptual categories: Vegetation, Open Smooth, Open Coarse, Rivers, Agrarian, and Structures. Both the Australian and American samples liked Rivers best and the Open categories least. Only the Australians included willow trees in the Agrarian category. The Australians liked the settings

AUTHORS'NOTE: We gratefully acknowledge the invaluable assistance of Mr. Mike Harper, formerly of the Department of Environmental and Natural Resources and now an officer of the Australian Landscape Trust, who arranged for all of the Australian respondent groups to participate in this study. Correspondence concerning this article should be addressed to Thomas R. Herzog, Department of Psychology, Grand Valley State University, Allendale, MI 49401; e-mail: herzogt@gvsu.edu.

ENVIRONMENT AND BEHAVIOR, Vol. 32 No. 3, May 2000 323-346

() 2000 Sage Publications, Inc. 
overall better than the Americans. Among the Australians, primary students liked the settings most, secondary students least; aboriginal college students liked the settings better than other college groups, but they disliked the Structures category; and DENR (Department of Environmental and Natural Resources) staff members liked the settings better than other Australian adults but, unlike other adults, did not like willows better than nonwillow settings. Cultural and evolutionary reasons for the complex pattern of results were explored.

One of the best ways to see the rich interplay of nature and nurture in environmental psychology is to make cross-cultural and subcultural comparisons of landscape perceptions and preferences. Nature, in the form of evolutionary predispositions, should show up in broad similarities across cultures that are consistent with evolutionary thinking. Nurture should appear primarily in cross-cultural and subcultural differences that can be traced to cultural, social, and other experiential influences.

Until about the middle of the 1980s, most cross-cultural comparisons of landscape preference consisted of cross-cultural correlations of preference ratings for the same sample of settings. Such correlations were typically high for similar cultures (e.g., Americans and Europeans or Australians) but lower for dissimilar cultures (e.g., Americans and West Indians) (Kaplan \& Herbert, 1987; Kaplan \& Kaplan, 1989). Kaplan and Herbert (1987) pointed out that correlations tell only part of the story. Comparisons of mean preference ratings can provide valuable additional insights. Likewise, stimulusgrouping procedures such as factor analysis permit comparison of perceptual categories based on preference ratings. Such comparisons allow us to see how different cultures categorize landscape settings.

Studies reviewed by Kaplan and Kaplan (1989) showed that each of these analytic approaches (correlations, comparison of means, and stimulus grouping) provides unique insights about cultural and subcultural similarities and differences. As noted earlier, correlational approaches show that relative liking for the same sample of settings tends to vary directly with the similarity of cultures. Means comparisons often (but not always) document a familiarity effect, with higher preference ratings from local citizens or cultures. Stimulus-grouping procedures typically yield broad cross-cultural agreement on basic features that define landscape categories (e.g., openness, smoothness of ground surface, presence of water, and signs of human influence), with differences primarily in the way these features are combined to form categories or in the specific settings composing categories.

The study by Kaplan and Herbert (1987) illustrated these points. They compared three groups: American and Australian college students and members of the wildflower society of Western Australia. The first two groups 
permitted a cross-cultural comparison, the last two groups a subcultural comparison. All groups provided preference ratings for 60 scenes of Western Australian forests and open areas. Correlations across groups were uniformly high (from .65 to .84), illustrating the similarity in the Australian and American cultures. Nonetheless, comparison of means and perceptual categories yielded interesting differences. Both Australian groups had somewhat higher mean ratings (3.32 on a 5-point scale for the wildflower society members, 3.28 for the students) than the American students (3.11), a familiarity effect. The subcultural comparison between the two Australian groups was also illuminating. The wildflower society members preferred native plants (e.g., eucalypt trees) to imported species (e.g., pines), but the students made no such distinction. Comparison of perceptual categories (derived from factor analysis) for the two student groups showed that categories tended to be based on similar considerations (openness, ground texture, and aridity) but that the Americans made finer distinctions (five categories versus three for the Australians).

Taking our cue from Kaplan and Herbert, we also compared Australian and American groups on preferences for Australian landscapes. There were two major differences from the previous study. First, our sample of settings was broader. Kaplan and Herbert concentrated on forests and open areas. We sampled six a priori landscape categories: rivers, dry lake beds, floodplains, terraces, mallee plains, and cultural images (including signs of former habitation and planned landscaping). For readers unfamiliar with the Australian landscape, mallee plains occupy the sand ridges or dunes above floodplains and are populated by short eucalypt trees. Second, and most important, our study examined several subcultural groupings within the Australian sample. The major subcultural grouping was based on age: primary, secondary, and college students; and adults. The Australian college students could be further subdivided: landscape architecture students, Australian aboriginal students, and regular students. Likewise, the Australian adults could also be subdivided: teachers, Lions club members, theater-group members, and members of the Department of Environmental and Natural Resources (DENR) staff. All groups at each level of analysis were mutually exclusive (i.e., no overlap in group or subgroup membership). Thus, we were able to make a much richer set of subcultural comparisons than is typically the case in landscape preference studies, and some of them (landscape architecture vs. other students and DENR staff vs. other adults) may be viewed as comparisons between experts and nonexperts. We were very excited at the prospect of reporting on the landscape preferences of aboriginal students, but our sample was quite small $(N=9)$ and thus all such results should be interpreted with great caution. 
There are hints in the literature about what to expect for some of our subcultural comparisons. Balling and Falk's (1982) study of the savanna hypothesis (a subset of the biophilia hypothesis) was based on an age-group comparison of preference for five biomes. The age groups consisted of students in grades three, six, and nine (i.e., 8-, 11-, and 15-year-olds), college students, adults, and retired citizens, all Americans. The 8-year-old group had the highest preference, and the 15-year-old group had the lowest preference. Thus, if Australians are similar to Americans in this regard, we might expect the highest preference ratings from our primary students and the lowest ratings from our secondary students. Meanwhile, several studies reviewed by Kaplan and Kaplan (1989) suggested that experts and nonexperts differ in landscape preferences. Perhaps the most dramatic is a study by Buhyoff, Wellman, Harvey, and Fraser (1978). In contrast to the usual high correlations between preference ratings by different groups, they found a near zero correlation between landscape architects and lay groups. Kaplan (1973) found that architecture majors liked wooded nature settings less and building complexes more than either landscape architecture or psychology majors. The landscape architecture majors liked a third category best, settings consisting partly of buildings and partly of landscaping. Studies such as these suggest that our experts (landscape architecture students and DENR staff) might have different patterns of preference from those of other subgroups.

In general then, our study examined cultural and subcultural similarities and differences in landscape perception and preference using a variety of analytic approaches: correlations, stimulus-grouping procedures, and mean comparisons. We used a very broad sample of landscapes and were able to make finer subcultural comparisons than is typically the case. Although the literature provides hints about what to expect for many of the comparisons (e.g., age comparisons and expert-nonexpert comparisons), some of the other comparisons are, to our knowledge, unprecedented (e.g., aboriginal vs. other students and teachers and theater-group members vs. other adults).

\section{METHOD}

\section{PARTICIPANTS}

The Australian sample consisted of 384 participants in four age groups: primary school students $(N=130)$, secondary school students $(N=79)$, college students $(N=68)$, and adults $(N=107)$. Both the primary and secondary student samples came from two different schools, with approximately equal 
subsamples from each school. The participating students were chosen by their teachers. The primary students represented only upper level primary school grades (ages 10 to 12), whereas the secondary students spanned all high school grades (ages 13 to 17). The majority of the college students (47) were landscape architecture students, but this subgroup also included 9 Australian aboriginals attending a college class dealing with environmental/park management and 12 students pursuing other majors. The college students were sampled from two different campuses. The adults included 52 teachers, 33 Lions club members, 14 members of the Department of Environmental and Natural Resources staff, and 8 members of a theater group. The teachers were sampled from all of the primary and secondary schools participating in the study plus attendees at the Riverland Teachers Conference. The DENR staff included professionals, field staff, and some office personnel, but no record of the number of participants within these categories was obtained. The American sample consisted of 250 undergraduate students enrolled in introductory psychology courses at Grand Valley State University (located in Western Michigan).

\section{STIMULI}

The settings were 60 color slides of natural environments in Australia. They were drawn primarily from the Bookmark Biosphere Reserve in Southeastern Australia and its surrounding region. The sample also included some cultural images depicting current and historical uses of the region. Six a priori landscape categories were equally represented in the slide set: rivers, dry lake beds, floodplains, terraces, mallee plains, and cultural. The dry lake beds were large open flat areas, generally devoid of vegetation, with very smooth ground surfaces stretching off into the distance. The floodplains were generally flat expanses of terrain surrounding rivers or lakes and containing a mixture of open grassland and eucalypt woodland. The terraces consisted of gently sloping terrain rising from floodplains to the mallee plains above. They were generally treeless with a shrub layer for ground cover. The mallee plains were dominated by multistemmed eucalypt trees approximately 5 meters in height. They occupy the sand ridges or dunes above the floodplains. The cultural category consisted of settings that displayed two kinds of obvious human influence: signs of former habitation (ruins, sheep troughs, and rehabilitation works) and planned agriculture (horticulture and terraforming for revegetation purposes). 
PROCEDURE

Participants were run in groups ranging in size between 8 and 42. All groups rated the 60 settings for preference, defined as how pleasing they found the setting, or how much they liked it. All ratings used a 5-point scale ranging from not at all to very much. The slides were shown in the same random order for all groups. The slides were preceded by three practice slides and followed by two filler slides. Participants were not told that the last two slides were fillers in hopes of avoiding end-of-task anticipation effects. The Australian groups had 10 seconds to view each slide, and the American groups had 15 seconds.'

\section{RESULTS}

Unless otherwise noted, analyses were based on settings as the units of analysis and setting scores as raw scores. A setting score is the mean preference rating for each setting based on all participants in the group being analyzed. With one exception (noted following), alpha was set at .05 for all tests of inference.

Our data-analytic strategy was first to examine correlations among the groups and subgroups based on setting scores. We then used factor analysis to derive empirical groupings of the settings separately for the American and combined Australian samples. This approach allowed a cross-cultural comparison of perceived landscape categories. As part of this comparison, we also examined whether the derived setting categories differed from each other in mean preference and whether there were any differences in mean preference between the Australian-based and American-based categories. We then proceeded to a series of four planned analyses focusing on specific questions regarding cross-cultural and subcultural comparisons of mean preferences. All of these analyses involved groups by setting categories analyses of variance. The first was a cross-cultural comparison involving the two comparable age groups of Australian and American college students. The remaining three analyses were subcultural comparisons involving only Australian groups: one comparing the four age groups, one comparing the three groups of Australian college students, and one comparing the four groups of adult Australians. We view all of the foregoing as focused planned analyses. Our final set of analyses was clearly post hoc and auxiliary, suggested by the results of the factor analyses. These analyses compared mean preferences for settings with and without willow trees for the various crosscultural and subcultural groups. 
TABLE 1

Reliability Coefficients (on diagonal) and Intergroup Correlations (above diagonal) for the Australian and American Age Groups

\begin{tabular}{lccccccc}
\hline Age Groups & 1 & 2 & 3 & 4 & 5 & 6 & $\mathrm{~N}$ \\
\hline 1. Australians & .99 & .96 & .99 & .97 & .93 & .91 & 384 \\
2. Primary students & & .99 & .95 & .94 & .81 & .93 & 130 \\
3. Secondary students & & .99 & .95 & .89 & .93 & 79 \\
4. College students & & & & .97 & .85 & .93 & 68 \\
5. Adults & & & & .99 & .73 & 107 \\
6. Americans & & & & & .99 & 250 \\
\hline
\end{tabular}

NOTE: Units of analysis for all coefficients are the 60 settings. Reliability coefficients are Cronbach's alphas. $N$ is the number of participants in each group.

\section{PREFERENCE CORRELATIONS}

Overall, the Americans and Australians showed a very high level of agreement in their preference ratings. The correlations of the 60 mean setting ratings for the major groups of this study are presented in Table 1 along with internal-consistency reliability coefficients (Cronbach's alpha) for the setting scores. The table shows that the Australian adults differed most from the other groups. When the Australian college students and adults are broken down further into subgroups, the correlations and reliabilities (not shown in Table 1) are somewhat lower. ${ }^{2}$ The correlations range down to .64 (between members of the Australian DENR and the American college students) but most are in the $.80 \mathrm{~s}$ and $.90 \mathrm{~s}$, with a few in the $.70 \mathrm{~s}$. The reliabilities all exceed .95 with the exception of the aboriginal students (.89), the regular Australian students (.93), the members of the theater group (.91), and the DENR members (.93). Overall then, the correlations were similar to those of past studies in indicating high agreement in relative landscape preferences between Australians and Americans.

\section{CROSS-CULTURAL PERCEPTION COMPARISON}

As noted in the introduction, a second useful approach to cross-cultural comparison is to examine empirically derived perceptual categories based on preference ratings. Such categories provide two very useful kinds of information. First, they allow us to probe for cross-cultural similarities and differences in the categories themselves. Second, they allow us to examine mean preference differences across the categories and to compare such differences across cultures and subcultures. 
TABLE 2

Correspondence Between the Australian and American Setting Categories

\begin{tabular}{|c|c|c|c|c|c|c|c|}
\hline & \multirow[b]{2}{*}{$\begin{array}{l}\text { Number } \\
\text { of Settings }\end{array}$} & \multicolumn{6}{|c|}{ Australian Categories } \\
\hline & & Vegetation & $\begin{array}{l}\text { Open } \\
\text { Smooth }\end{array}$ & $\begin{array}{l}\text { Open } \\
\text { Coarse }\end{array}$ & Rivers & Agrarian & Structures \\
\hline Number of settings & & 20 & 11 & 7 & 5 & 5 & 3 \\
\hline \multicolumn{8}{|l|}{ American categories } \\
\hline Vegetation & 13 & 12 & & & & 1 & \\
\hline Open Smooth & 6 & & 6 & & & & \\
\hline Open Coarse & 14 & 3 & 2 & 6 & & & \\
\hline Rivers & 9 & 1 & & & 5 & 1 & \\
\hline Agrarian & 5 & & 1 & 1 & & 3 & \\
\hline Structures & 5 & & & & & & 3 \\
\hline
\end{tabular}

The perceptual categories were derived by means of nonmetric factor analysis of preference ratings (i.e., smallest space analysis III) (Lingoes, 1972). Such analysis was carried out separately for the American and the combined Australian samples so that their perceived landscape categories could be compared. It was not considered advisable to do separate factor analyses for each Australian subgroup because of the unacceptably small sample sizes for the subgroups $(N \leq 130)$. For both the American and Australian factor analyses, solutions in several dimensions were examined. The criterion for category membership was that a setting had to have a factor loading of at least $|.40|$ on one dimension only. With that criterion, it was clear that there were six categories for both the Australians and the Americans and that the categories were substantially the same in both cases. Even so, there was a fair degree of nonoverlap in category composition between the two cultures, as described next. Table 2 shows the correspondence between the Australianand American-based categories. Figures 1 through 6 show examples of settings that appeared in both the Australian and American versions of each setting category.

The largest category had 12 settings common to both samples. These all featured good-sized trees or bushes in the near or relatively near distance. The a priori categories were represented as follows: six mallee plains, five floodplains, and one lake bed. The American version of this category also included a river setting, but one in which the water was not readily visible and riverside trees were. The Australian version of this category had eight additional settings: four mallee plains, two floodplains, a terrace, and a lake bed. Each of the settings in this category had its defining feature, visible good-sized vegetation, although in some of the low-loading settings of the Australian version 


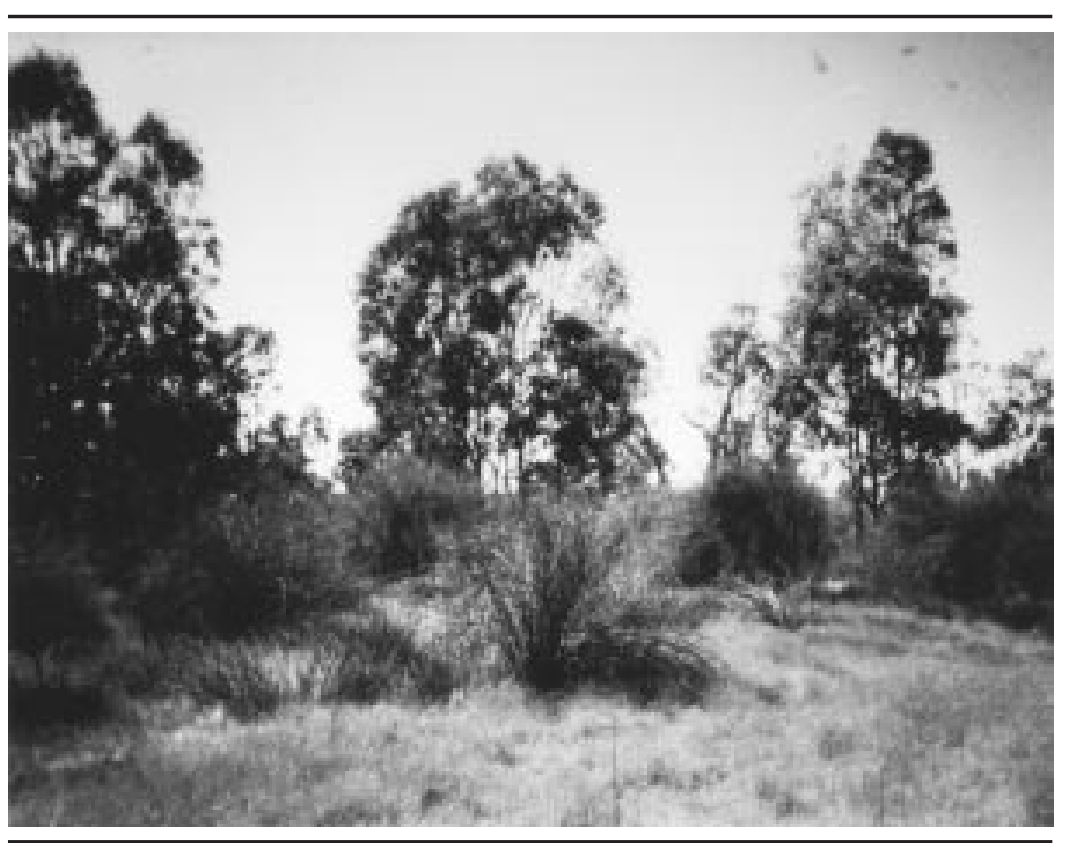

Figure 1: Setting in the Vegetation Category

there was relatively little vegetation or it was off in the distance. Nonetheless, based on the great majority of the settings, this category seemed to be about prominent Vegetation.

The second category had six settings common to both samples, five dry lake beds, and a floodplain. These settings contrast sharply with those in the Vegetation category. In these settings, there is very little vegetation, mostly off in the far distance, and there is typically a very smooth foreground surface texture (the dry lake bed effect) stretching off into the distance. These settings commonly have little in the way of depth structuring. The Americans had no additional settings in this category, but the Australians had five: one lake bed, one real lake shore beside a bona fide lake, two terraces, and one agrarian landscape from the a priori cultural category. The two terraces lacked the smooth foreground texture typical of this category; their foregrounds were covered with scrub brush. The agrarian landscape was manicured in such a way that it had fairly prominent stretches of smooth foreground surface. It seems clear that the Australian version of this category was not as tightly focused as the American version. We think of this category as Open Smooth. 
332 ENVIRONMENT AND BEHAVIOR / May 2000

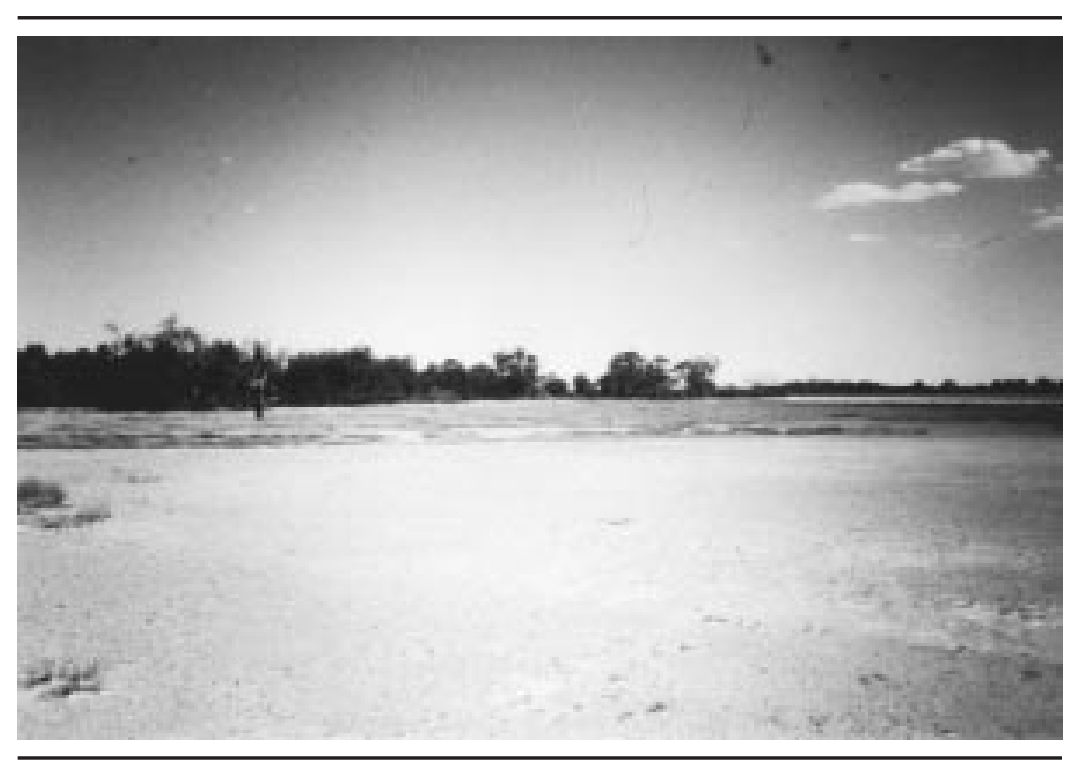

Figure 2: Setting in the Open Smooth Category

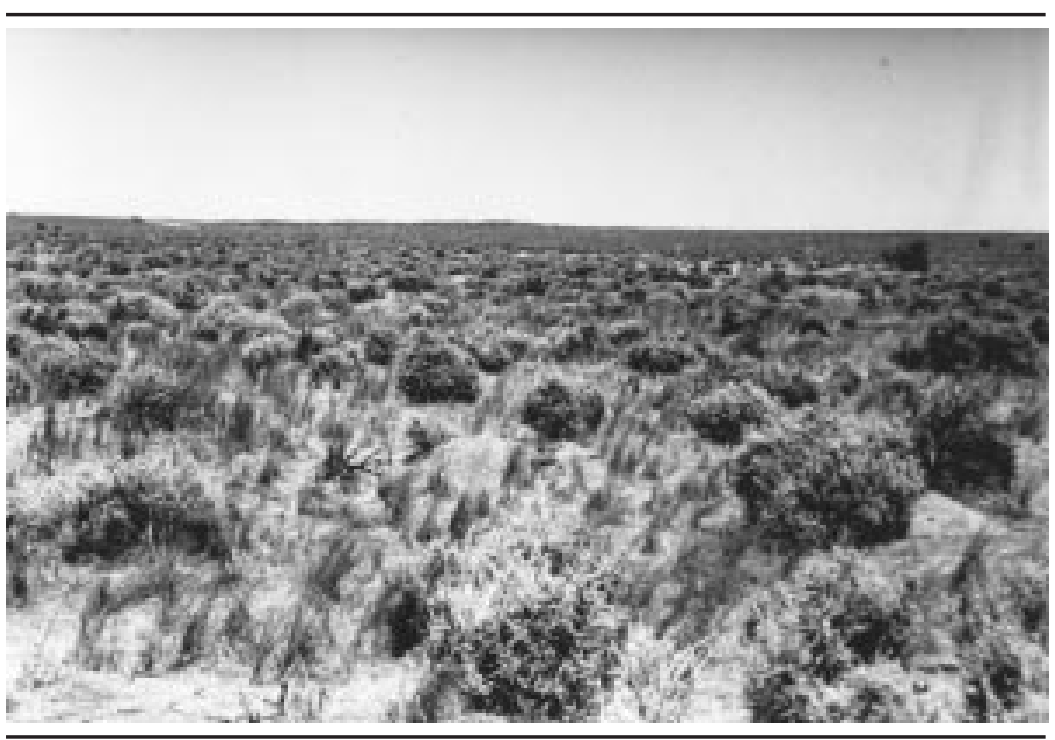

Figure 3: Setting in the Open Coarse Category 


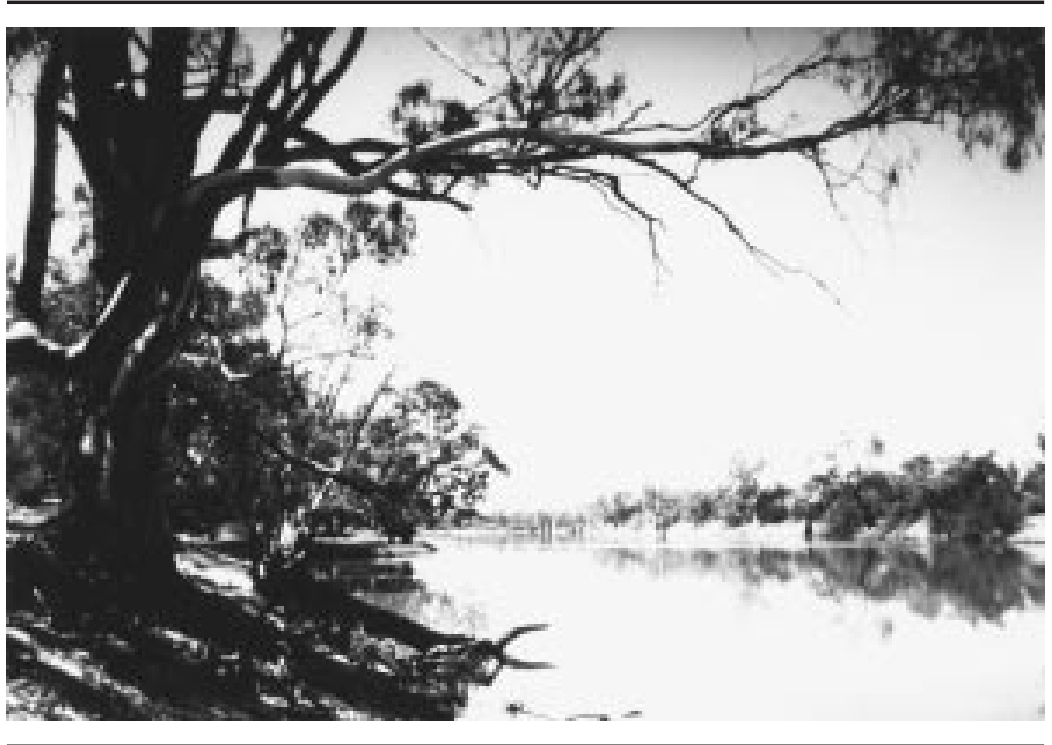

Figure 4: Setting in the Rivers Category

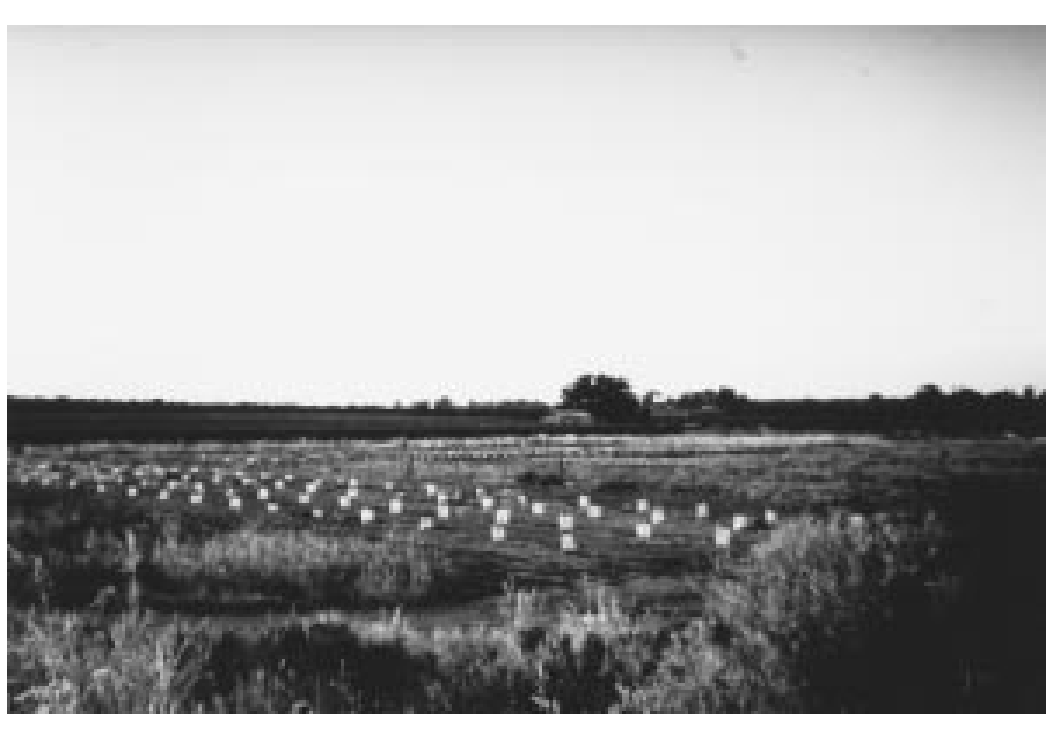

Figure 5: Setting in the Agrarian Category 


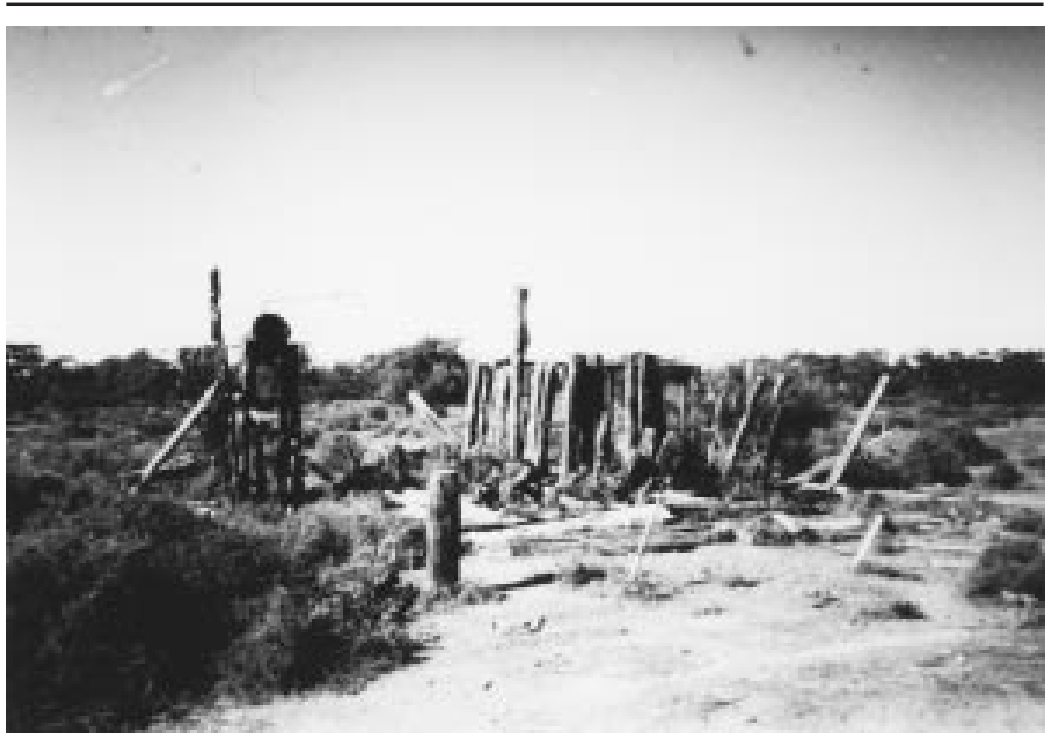

Figure 6: Setting in the Structures Category

The third category also had six settings common to both samples, five terraces and a lake bed. Similar to the Open Smooth category, this category also features very open settings with long views and little depth structuring. The contrasting feature is that the ground surface in these settings is typically covered with low scrub brush. The Australian version of this category contained one additional setting, a clearly manipulated agrarian landscape from the a priori cultural category. The American version of this category contained eight additional settings: three floodplains, three terraces, one mallee plain, and one lake bed. Of the settings unique to the Americans, three of them, two floodplains and a terrace, had the defining features of this category (long open view and scrub-brush ground cover) but also had visible trees in the distance. Indeed, one of these settings appeared in the Australian version of the Vegetation category. Finally, the Americans also had a floodplain in this category that contained a very smooth ground surface stretching into the far distance, making the setting look more like a lake bed than a floodplain. However, the setting also contained clearly visible trees in the far distance. This setting, with its mixed characteristics, appeared in no other solution. In general then, this category featured long views, little depth structuring, and scrub-brush ground cover. We called it Open Coarse. 
The fourth category was easy to define. The five settings common to both samples were all rivers. The Australians had no additional settings, but the Americans had four, three rivers and a floodplain. The floodplain was an anomaly, pure and simple. This category is Rivers.

The last two categories are human-influence categories. One represents human influence on the landscape, whereas the other consists of structural remnants. These categories represent the two subdivisions of the a priori cultural category. The first of these categories had three settings common to both samples, all agrarian landscapes. The Americans had two additional agrarian landscapes, but the Australians had two additional settings from the a priori Rivers category instead. Both of these settings contained clearly visible willow trees-a nonnative plant species and thus seen as a sign of human influence. One of these two settings had clearly visible water; the other did not. Taking our cue from the majority of the settings, we called this category Agrarian. The last category also had three settings common to both samples, all structural ruins. The Australian version of this category had no additional settings, but the American version had two, another ruin and a water trough. We called this category Structures.

It seems clear that there is a great deal of similarity in the features that define landscape categories for these two cultures: prominence of vegetation, openness, depth structuring, character of the ground surface, presence of water, and signs of human influence. These are just the features that have repeatedly been found to define perceptual categories in landscape preference research, and so their influence in this study is hardly surprising. Despite the strong similarity between the two cultures in what defines perceptual categories, there were differences in the specific settings within the categories as described earlier. One of these differences is readily interpretable. What defines an intrusive landscape element, a sign of human influence, depends on local experience. For Australians, willow trees are a clear sign of human influence; for Americans, they are not.

Do these perceptual categories differ in preference, and are there any preference differences associated with the Australian and American versions of the categories? The answer to the first question is a strong yes. Collapsed across sample groups and both versions of the categories, the mean preference ratings for the six setting categories differed significantly, $F(5,91)=$ $38.08, p<.001$; partial eta squared $=.68 .^{3}$ The most preferred category was Rivers $($ mean $=4.04)$. Significantly lower in preference and not different from each other (as indicated by pairwise post hoc comparisons using the Tukey-B test) ${ }^{4}$ were the Agrarian (3.08) and Vegetation (3.02) categories. The final step down consisted of the remaining three categories, all not differing from each other and significantly lower than the second tier just 
described. The rank order of mean preferences was Structures (2.57), Open Smooth (2.40), and Open Coarse (2.28).

To detect preference differences associated with the Australian and American versions of the setting categories, we tested the simple main effect of version for each category. Because there were six tests in the set, alpha was lowered to $.05 / 6=.008$ for these tests. The version made a difference only for the Agrarian category, $F(1,91)=7.88, p<.007$; partial eta squared $=.08$. The Australian version of that category was higher in preference than the American version (means of 3.44 and 2.73, respectively). This difference must be due to the two settings that are unique to each version of the category. The unique settings in the Australian version are the two willow settings described earlier. Their mean preference was 3.96. The unique settings in the American version of the category, two agrarian settings, had a mean preference of 2.21. Thus, although the willows were perceived by the Australians as signs of human intrusion, the overall preference for these settings was quite high.

In summary, nonmetric factor analysis revealed the same six perceptual categories for both the Australian and American samples: Vegetation, Open Smooth, Open Coarse, Rivers, Agrarian, and Structures. There were slight differences in the specific settings within the categories for the two samples, the most notable being the inclusion of the two settings containing willow trees in the Agrarian category only for the Australian sample. The categories differed in overall preference, with Rivers highest and the Open categories and Structures lowest. The Australian and American versions of the setting categories differed in mean preference only for the Agrarian category. The higher preference for the Australian version of that category is clearly attributable to its unique inclusion of the two settings containing willow trees.

\section{CROSS-CULTURAL PREFERENCE COMPARISON}

Although the entire Australian sample was used for the factor analysis, the cross-cultural comparison of means is most appropriately based on the college student groups from both cultures. These two groups provide the cleanest cross-cultural comparison possible because they are equated for age and level of education. Because each group can be compared using both the Australian and American versions of the setting categories, the three independent variables were college group (Australian and American), setting categories, and version of the setting categories (Australian based and American based). The main focus here is on effects involving college group, of which two were significant. Overall, the Australian college students had higher preference ratings than the Americans, means of 3.01 and 2.76 , respectively $-F(1,91)=$ 
TABLE 3

Mean Preferences of College-Student Groups as a Function of Setting Category

\begin{tabular}{lcccccc}
\hline & \multicolumn{6}{c}{ Setting Category } \\
\cline { 2 - 7 } College Group & $\begin{array}{c}\text { Vegetation } \\
(\mathrm{N}=33)\end{array}$ & $\begin{array}{c}\text { Smooth } \\
(\mathrm{N}=17)\end{array}$ & $\begin{array}{c}\text { Open } \\
\text { Coarse }\end{array}$ & $\begin{array}{c}\text { Rivers } \\
(\mathrm{N}=21)\end{array}$ & $\begin{array}{c}\text { Agrarian } \\
(\mathrm{N}=14)\end{array}$ & $\begin{array}{c}\text { Structures } \\
(\mathrm{N}=10)\end{array}$ \\
\hline Australian & 3.18 & 2.51 & 2.39 & 4.00 & 3.25 & 2.94 \\
American & 2.78 & 2.47 & 2.23 & 3.88 & 3.07 & 2.36 \\
\hline
\end{tabular}

NOTE: $N$ is the number of settings on which each mean in the column is based.

$131.37, p<.001$; partial eta squared $=.59$-illustrating the expected familiarity bias for settings from their own country. However, the preference advantage of the Australians differed across setting categories, $F(5,91)=14.38, p<$ .001 ; partial eta squared $=.44$. This interaction is presented in Table 3. Post hoc tests showed that the two groups did not differ for the Open Smooth and Rivers categories but did differ for the other four categories.

\section{SUBCULTURAL PREFERENCE COMPARISONS}

Age groups. Subcultural comparisons were possible only within the Australian sample. That being the case, we used only the Australian version of the setting categories in these analyses. The first subcultural variable to be examined was the age grouping. The age groups differed in overall preference, $F(3,135)=39.56, p<.001$; partial eta squared $=.47$. Post hoc tests showed that the primary students had the highest mean preference (3.22), the secondary students the lowest (2.79), with the other two groups in between and not different from each other (means of 2.98 and 3.00 for the adults and college students, respectively). However, the overall ordering of the age groups varied with the setting category, $F(15,135)=15.24, p<.001$; partial eta squared $=.63$. This interaction is illustrated in Table 4 . The adults were the most variable in their relative preferences. Post hoc tests confirmed that they were alone at the top of the ordering of groups in the Vegetation category and tied for top spot in the Open Coarse and Rivers categories but they were alone in last place in the Open Smooth category and tied for last in the Agrarian category. The other noteworthy feature of Table 4 is that the primary students were always at or near the top of the rank ordering of groups and the secondary students near the bottom (with one exception: Rivers). 
TABLE 4

Mean Preferences of Australian Participants as a Function of Age Group and Setting Category

\begin{tabular}{lcccccc}
\hline & \multicolumn{7}{c}{ Setting Category } \\
\cline { 2 - 7 } Age Group & $\begin{array}{c}\text { Vegetation } \\
(\mathrm{N}=20)\end{array}$ & $\begin{array}{c}\text { Smooth } \\
(\mathrm{N}=11)\end{array}$ & $\begin{array}{c}\text { Open } \\
\text { Coarse } \\
(\mathrm{N}=7)\end{array}$ & $\begin{array}{c}\text { Rivers } \\
(\mathrm{N}=5)\end{array}$ & $\begin{array}{c}\text { Agrarian } \\
(\mathrm{N}=5)\end{array}$ & $\begin{array}{c}\text { Structures } \\
(\mathrm{N}=3)\end{array}$ \\
\hline Primary students & 3.28 & 2.81 & 2.30 & 4.44 & 3.91 & 3.24 \\
Secondary students & 2.89 & 2.19 & 1.99 & 4.38 & 3.33 & 2.62 \\
College students & 3.06 & 2.50 & 2.37 & 4.03 & 3.64 & 3.14 \\
Adults & 3.44 & 1.96 & 2.31 & 4.31 & 3.11 & 2.81 \\
\hline
\end{tabular}

NOTE: $N$ is the number of settings on which each mean in the column is based.

College student groups. The three Australian college student groups differed in overall preference, $F(2,90)=22.14, p<.001$; partial eta squared $=$ .33. Post hoc tests showed that all three groups differed from each other, with the aboriginal students having the highest mean preference (3.25), the regular students the lowest (2.72), and the landscape architecture students in between (3.03). However, the ranking of groups varied with the setting category, $F(10$, $90)=11.09, p<.001 ;$ partial eta squared $=.55$. This interaction is illustrated in Table 5. Post hoc tests showed that the aboriginal students were alone with the highest preference in the Vegetation and Agrarian categories and shared first place in all the remaining categories except Structures, where notably, they were alone in last place. The landscape architecture students were alone in first place for Structures and shared the top spot in the Open categories but they were alone in last place for Rivers. The regular students were either alone in last place (Vegetation and both Open categories) or shared it in all categories except Rivers, where they shared top billing with the aboriginal students.

Adult groups. The four Australian adult groups differed in overall preference, $F(3,135)=23.16, p<.001$; partial eta squared $=.34$. Post hoc tests showed that the DENR staff had the highest mean preference (3.23), the teachers the lowest (2.85), with the other two groups in between and not different from each other (means of 3.01 and 3.08 for the theater-group members and Lions club members, respectively). The ordering of the adult groups differed somewhat across setting categories, $F(15,135)=3.94, p<.001$; partial eta squared $=.30$. This weak interaction is illustrated in Table 6 . The variations from the overall pattern were slight, the most striking occurring in the Agrarian category. That was the only category in which the DENR staff 
TABLE 5

Mean Preferences of Australian College-Student Groups as a Function of Setting Category

\begin{tabular}{lcccccc}
\hline & \multicolumn{7}{c}{ Setting Category } \\
\cline { 2 - 7 } College Group & $\begin{array}{c}\text { Vegetation } \\
(\mathrm{N}=20)\end{array}$ & $\begin{array}{c}\text { Open } \\
(\mathrm{N}=11)\end{array}$ & $\begin{array}{c}\text { Open } \\
\text { Coarse } \\
(\mathrm{N}=7)\end{array}$ & $\begin{array}{c}\text { Rivers } \\
(\mathrm{N}=5)\end{array}$ & $\begin{array}{c}\text { Agrarian } \\
(\mathrm{N}=5)\end{array}$ & $\begin{array}{c}\text { Structures } \\
(\mathrm{N}=3)\end{array}$ \\
\hline Aboriginal students & 3.44 & 2.49 & 2.54 & 4.53 & 4.33 & 2.37 \\
Landscape students & 3.07 & 2.62 & 2.48 & 3.86 & 3.49 & 3.38 \\
Regular students & 2.76 & 2.05 & 1.81 & 4.33 & 3.72 & 2.75 \\
\hline
\end{tabular}

NOTE: $N$ is the number of settings on which each mean in the column is based.

was not either first in the ranking or sharing the top spot and in which the teachers were not last or tied for last.

In summary, comparison of Australian age groups revealed that the primary students had the highest overall preference and the secondary students the lowest. The adults were quite variable across the setting categories in their preference ranking relative to the other age groups. Comparison of Australian college student groups revealed that the aboriginal students had the highest overall preference and the regular college students had the lowest. However, the aboriginals had the lowest preference for the Structures category, and the landscape students had the highest preference. The regular college students had the same high level of preference for the Rivers category as did the aboriginal students. Comparison of the Australian adult groups revealed that the DENR staff had the highest overall preference and the teachers had the lowest. The Agrarian category was the only one that did not follow this pattern.

\section{WILLOWS}

Earlier we noted that the major difference between the Australian and American versions of the setting categories occurred in the Agrarian category where the Australian version of the category was significantly higher in preference than the American version. This appeared to be due at least in part to the fact that the only two settings among the 60 scenes that contained willow trees appeared only in the Australian version of the Agrarian category. Because these two willow settings were highly preferred (mean $=3.96)$, the Australian version of the category benefited uniquely from a preference boost. Given this unexpected role of willow settings in our results, we considered it worthwhile to explore the role of willows further in a set of auxiliary 
TABLE 6

Mean Preferences of Australian Adults as a Function of Setting Category

\begin{tabular}{lcccccc}
\hline & \multicolumn{7}{c}{ Setting Category } \\
\cline { 2 - 7 } Adult Group & $\begin{array}{c}\text { Oegetation } \\
(\mathrm{N}=20)\end{array}$ & $\begin{array}{c}\text { Opmooth } \\
(\mathrm{N}=11)\end{array}$ & $\begin{array}{c}\text { Open } \\
\text { Coarse } \\
(\mathrm{N}=7)\end{array}$ & $\begin{array}{c}\text { Rivers } \\
(\mathrm{N}=5)\end{array}$ & $\begin{array}{c}\text { Agrarian } \\
(\mathrm{N}=5)\end{array}$ & $\begin{array}{c}\text { Structures } \\
(\mathrm{N}=3)\end{array}$ \\
\hline Theater group & 3.46 & 2.08 & 2.38 & 4.42 & 2.70 & 3.04 \\
Lions club members & 3.44 & 1.99 & 2.55 & 4.50 & 3.40 & 3.05 \\
Teachers & 3.36 & 1.83 & 2.08 & 4.13 & 3.03 & 2.47 \\
DENR staff & 3.74 & 2.29 & 2.57 & 4.44 & 2.93 & 3.36 \\
\hline
\end{tabular}

NOTE: $N$ is the number of settings on which each mean in the column is based. $D E N R=$ Department of Environmental and Natural Resources.

analyses. In these analyses, we contrasted preference for the willow settings with preference for all the rest of the settings, labeling this effect willowness. Because of the extremely small number of willow settings (two), our results for willowness should be considered suggestive at best. We did a series of four analyses in which willowness and sample groups were independent variables. The analyses differed in how sample groups were defined. In one analysis, sample groups referred to the Americans versus all Australians, and in the other three analyses, it referred to the three subcultural groupings of the Australian sample (by age, by college student groups, and by adult groups). These analyses allowed us to see if willowness interacted with any of our sample grouping variables.

The results were that the main effect of willowness was significant $(p<$ .05 ) in all analyses except the one for the Australian adult groups. Willow settings were always preferred over nonwillow settings, the largest difference occurring for the Australian college students (mean preferences of 4.39 vs. 2.97 ) and the smallest difference for the Australian adults (means of $3.62 \mathrm{vs}$. 3.04). Effect sizes were always small (largest partial eta squared $=.11$ for the college students). We were primarily interested in the interaction of willowness with the sample-grouping variable. That interaction was significant in two of the analyses, the one for college students, $F(2,116)=4.23, p<.02$; partial eta squared $=.07$; and the one for adults, $F(3,174)=8.14, p<.001$; partial eta squared $=.12$. Both interactions are shown in Table 7. For the college students, post hoc tests showed that the willowness effect was significant for all three groups. However, inspection reveals that the effect was smaller for the expert group (landscape students) than for the other groups. The same tendency toward expert-nonexpert differences appeared more dramatically 
TABLE 7

Mean Preferences of Australian College-Student and Adult Groups as a Function of Willowness

\begin{tabular}{|c|c|c|}
\hline \multirow[b]{2}{*}{ Sample Group } & \multicolumn{2}{|c|}{ Willowness } \\
\hline & $\begin{array}{c}\text { No } \\
(\mathrm{N}=58)\end{array}$ & $\begin{array}{c}\text { Yes } \\
(\mathrm{N}=2)\end{array}$ \\
\hline \multicolumn{3}{|l|}{ College group } \\
\hline Aboriginal students & 3.20 & 4.78 \\
\hline Landscape students & 3.02 & 3.89 \\
\hline Regular students & 2.69 & 4.50 \\
\hline \multicolumn{3}{|l|}{ Adult group } \\
\hline Theater group & 2.98 & 3.62 \\
\hline Lions club members & 3.08 & 4.06 \\
\hline Teachers & 2.85 & 3.66 \\
\hline DENR staff & 3.26 & 3.14 \\
\hline
\end{tabular}

\section{DISCUSSION}

As noted in the introduction, cross-cultural and subcultural comparisons provide a forum for appreciating the rich interplay of nature and nurture in environment-behavior interactions. In the current data, as in many past studies, one can make a strong case for a significant role of nature in the defining features of the perceptual categories found across cultures. These features include environmental attributes such as openness, ground texture, water, vegetation, and signs of human intrusion, all of which have been linked to evolutionary predispositions (Kaplan \& Kaplan, 1989). At the extreme other end of the continuum, variations in overall preference reactions, as illustrated by several of the interactions involving sample groups, are often most readily explained in terms of nurture, that is, cultural, social, and other experiential influences. Prime examples are the preference differences between experts (such as DENR staff and landscape architecture students) and nonexperts that occur only in certain setting categories. Meanwhile, the great bulk of the findings can be attributed without favoritism to both nature and nurture. For example, that all groups prefer rivers seems likely to be due both to 
evolutionary predispositions favoring water and to strong cross-cultural biases favoring most waterscapes.

Still, at a general level, our study illustrates again (as did Kaplan \& Herbert, 1987) the usefulness of a three-pronged analytic approach to crosscultural comparisons: correlations (the traditional approach), stimulus grouping into perceptual categories, and comparison of means. Each of these approaches provides unique insights and enriches understanding. Perhaps the clearest example of this (but only one of many that could be cited) is in our findings for settings containing willow trees. As individual settings, they were highly preferred and helped anchor the high preference end of the cross-groups preference correlations. As members of perceptual categories, however, willows produced cross-cultural differences. For the Australians, they are a nonnative species, indicative of human manipulation of the landscape, and thus were grouped into the Agrarian category. For the Americans, this was not so. Finally, comparison of means provided yet a different perspective. Whether or not they were perceived as signs of human intrusion, the willows were generally highly preferred by all groups, with the notable exception of DENR staff members, about which more follows.

In this study, it was certainly true that cross-cultural similarities were far greater in magnitude than any of the differences we found. With an overall correlation of .91 between setting scores for the American and combined Australian samples (Table 1), any differences between the two cultures in our subsequent analyses had to be modest in magnitude. Three comments about this disparity in effect sizes are in order. First, the cross-cultural similarity was to be expected given the known similarity between the two cultures, as outlined in the introduction. Second, the cultural and subcultural differences we found are no less interesting because they are small in magnitude. Third, the method that produced the current disparity in effect sizes (the threepronged approach) should prove very useful in examining less similar cultures.

Many of our findings fit well with past research on environmental preferences. One example is the familiarity bias, the greater liking for Australian landscapes by Australians as compared to Americans. A variation on this theme was the greater overall preference by Australian aboriginal students compared to other Australian students. Although caution is called for because of the small aboriginal sample, this difference is not surprising. The aboriginals presumably have closer ties to the Australian landscape than other Australian citizens, and there is currently a great deal of controversy in Australia regarding native title to traditional lands. Yet, as Kaplan and Kaplan (1989) pointed out, familiarity does not always enhance preference. The 
aboriginals are presumably no less familiar with structural remnants in the landscape than are nonaboriginals, and yet the aboriginals disliked the Structures category. We can speculate that the images in this category reminded them of European invasion and their forced removal from traditional lands and subsequent persecution.

A second finding that fits the existing literature is the age trends in the current results. Similar to Balling and Falk (1982), we found that the primary students had the highest preference and the secondary students the lowest. The Australian and American cultures are apparently similar in this respect. A new wrinkle in our results was the evidence that the adults differed from the other age groups, not so much in overall preference but rather in lower correlations with the other age groups and greater variability in their mean preferences across setting categories. It seems reasonable to view this as the end result of a developmental sequence in which increasing maturity ultimately leads to a distinctive pattern of preferences. Balling and Falk (1982) did not present intergroup correlations, but their setting category means for adults did not show as much variability relative to other age groups as was the case in our data. This was probably due to the relatively restricted range of settings in their biome-based setting categories. Balling and Falk's settings included no waterscapes and no human-influenced landscapes. The development of a distinctive adult pattern of preferences may become more evident only when a sufficient variety of setting types is sampled.

The clearest age trend in both data sets was the high preference of the primary students and the low preference of the secondary students. Balling and Falk (1982) saw primary students as viewing the natural landscape through rose-colored glasses, primarily as a place to play and have a good time rather than the more adult vision as a place to eke out a living. This seems reasonable. There is evidence that children prefer adventure playgrounds to more traditional ones because of the freedom for self-expression afforded by adventure playgrounds (Hayward, Rothenberg, \& Beasley, 1974). From that perspective, primary students might well view natural landscapes as the best of all possible adventure playgrounds. Meanwhile, the shift of interest among secondary students to more social concerns (clothes, appearance, music, and relationships with peers) is well-known in the literature on adolescence (e.g., Santrock, 1998). Interest in the natural environment, similar to many other nonsocial interests, is uncool. Here, too, Australian and American youth are similar. It is encouraging to note that these developmental fluctuations result in a discriminating adult.

A third area in which our results fit well with existing literature and theory is in the data on differences between experts and nonexperts. Our 
comparisons involved landscape architecture students versus other college students and DENR staff members versus other adults. We found, as did Kaplan (1973), that the landscape architecture students liked structural remnants in a natural setting better than did other students. Although the landscape architecture students liked the Rivers category less than did the other two student groups, Rivers was nonetheless their most preferred category, as was true for each of the samples in the study. We also found that DENR staff members, who have an intensive and policy-setting involvement with the natural environment, tend to like natural landscapes better than other adults. There is very likely a self-selection bias operating here: People who choose to work for such an agency have a love for natural resources and their conservation.

Finally, to paraphrase Sherlock Holmes, what about the curious incident of the DENR staff members and the willows? Similar to the dog that did nothing in the nighttime, the DENR staff had no special liking for the willows in contrast to the other adult groups. What at first may seem mysterious becomes elementary (dear Watson) given a bit of local cultural history. The staff at DENR launched a campaign to eradicate willows, which they had classified as a noxious weed, from their local environment. They were well into the implementation of a poisoning scheme when the public noticed the disappearing willows and raised an outcry. This led to a debate between the experts (DENR) and the public (who claimed that they had not been consulted) that was widely publicized in the media. Eventually, the government stepped in and halted the poisoning program. As to the practical implications of expert-nonexpert differences in landscape perception and preference, we rest our case.

\section{NOTES}

1. Previous research (e.g., Herzog, 1984, 1985, 1987, 1989; Kaplan, 1975) suggested that these are both very long viewing times and are unlikely to produce any differences in ratings.

2. A reviewer noted that the smaller correlations for some of the subgroups could be a function of the smaller sample of raters for those groups $(N<20$ for the aboriginal and the regular college students and for the DENR staff and the members of the adult theater group). The small rater samples should lead to more random error in the setting scores, thus lowering correlations for those groups.

3. Partial eta squared is a measure of effect size. It is equal to the sum of squares for the effect divided by the sum of squares for the effect plus the sum of squares for the effect's error term. According to Hays (1973), eta-type measures overestimate the effect size for the population. We 
report partial eta squared because it is provided by SPSS, the statistical package used for data analysis.

4. We used Kramer's (1956) modification for unequal sample sizes. In our later post hoc analyses of significant interactions, we also used Cicchetti's (1972) modification for interaction tables. A reviewer pointed out that the Tukey-B test may not be appropriate when variances are not homogeneous across conditions. In all cases of significant results involving more than two means, we checked for homogeneity of variance using the default test in SPSS, Levene's test. For interaction tables, the test is automatically done separately at each level of a within-subjects independent variable. Because we used setting scores as our dependent variable, our sample grouping variables were always within-subjects (i.e., within-settings) independent variables. Levene's test was performed a total of 21 times. With alpha at .05, two of the tests were significant. However, both significant results involved interaction tables. After applying a Bonferroni correction for the number of tests within each interaction table, neither of these tests remained significant. Based on these tests for homogeneity of variance, we are confident that the Tukey-B is an appropriate post hoc test for our data.

\section{REFERENCES}

Balling, J. D., \& Falk, J. H. (1982). Development of visual preference for natural environments. Environment and Behavior, 14, 5-28.

Buhyoff, G. J., Wellman, J. D., Harvey, H., \& Fraser, R. A. (1978). Landscape architects' interpretations of people's landscape preferences. Journal of Environmental Management, 6 , 255-262.

Cicchetti, D. V. (1972). Extension of multiple-range tests to interaction tables in the analysis of variance: A rapid approximate solution. Psychological Bulletin, 77, 405-408.

Hays, W. L. (1973). Statistics for the social sciences. New York: Holt, Rinehart \& Winston.

Hayward, D. G., Rothenberg, M., \& Beasley, R. R. (1974). Children's play and urban playground environments: A comparison of traditional, contemporary, and adventure playground types. Environment and Behavior, 6, 131-168.

Herzog, T. R. (1984). A cognitive analysis of preference for field-and-forest environments. Landscape Research, 9, 10-16.

Herzog, T. R. (1985). A cognitive analysis of preference for waterscapes. Journal of Environmental Psychology, 5, 225-241.

Herzog, T. R. (1987). A cognitive analysis of preference for natural environments: Mountains, canyons, deserts. Landscape Journal, 6, 140-152.

Herzog, T. R. (1989). A cognitive analysis of preference for urban nature. Journal of Environmental Psychology, 9, 27-43.

Kaplan, R. (1973). Predictors of environmental preference: Designers and "clients.” In W.F.E. Preiser (Ed.), Environmental design research (pp. 265-274). Stroudsburg, PA: Dowden, Hutchinson, \& Ross.

Kaplan, R. (1975). Some methods and strategies in the prediction of preference. In E. H. Zube, R. O. Brush, \& J. G. Fabos (Eds.), Landscape assessment: Values, perceptions, and resources (pp. 118-129). Stroudsburg, PA: Dowden, Hutchinson, \& Ross.

Kaplan, R., \& Herbert, E. J. (1987). Cultural and sub-cultural comparisons in preferences for natural settings. Landscape and Urban Planning, 14, 281-293. 


\section{ENVIRONMENT AND BEHAVIOR / May 2000}

Kaplan, R., \& Kaplan, S. (1989). The experience of nature: A psychological perspective. New York: Cambridge University Press.

Kramer, C. Y. (1956). Extension of multiple range tests to group means with unequal numbers of replications. Biometrics, 12, 307-310.

Lingoes, J. C. (1972). A general survey of the Guttman-Lingoes nonmetric program series. In R. N. Shepard, A. K. Romney, \& S. B. Nerlove (Eds.), Multidimensional scaling (Vol. 1, pp. 52-68). New York: Seminar Press.

Santrock, J. W. (1998). Adolescence (7th ed.). New York: McGraw-Hill. 